\title{
Sub-10 nm Platinum Nanocrystals with Size and Shape Control: Catalytic Study for Ethylene and Pyrrole Hydrogenation
}

Chia-Kuang Tsung, John N. Kuhn, Wenyu Huang, Cesar Aliaga, Ling-I Hungt, Gabor A. Somorjai*, Peidong Yang*,

Department of Chemistry, University of California, Berkeley, California 94720 and Chemical and Materials Sciences Divisions, Lawrence Berkeley National Laboratory, 1 Cyclotron Road, Berkeley, California 94720 †Materials and Chemical Laboratories, Industrial Technology Research Institute, 195, Sec. 4, Chung Hsing Road, Chutung, Hsinchu, 310, Taiwan

\section{ABSTRACT}

Platinum nanocubes and nanopolyhedra with tunable size from $5 \mathrm{~nm}$ to $9 \mathrm{~nm}$ were synthesized by controlling the reducing rate of metal precursor ions in a one-pot polyol synthesis. A two-stage process is proposed for the simultaneous control of size and shape. In the first stage, the oxidation state of the metal ion precursors determined the nucleation rate and consequently the number of nuclei. The reaction temperature controlled the shape in the second stage by regulation of the growth kinetics. These well-defined nanocrystals were loaded into MCF-17 mesoporous silica for examination of catalytic properties. Pt loadings and dispersions of the supported catalysts were determined by elemental analysis (ICP-MS) and $\mathrm{H}_{2}$ chemisorption isotherms, respectively. Ethylene hydrogenation rates over the Pt nanocrystals were independent of both size and shape and comparable to Pt single crystals. For pyrrole hydrogenation, the nanocubes enhanced ring opening ability and thus showed a higher selectivity to $n$-butylamine compared to nanopolyhedra.

\section{Introduction}

The most important aim of heterogeneous catalysis is to design catalysts with high selectivity for the desired product without sacrificing activity. Transition metal 
nanocrystals with size and shape control are very promising for this goal. For example, alkane hydrogenolysis rates showed sensitivity to Pt nanocrystals size. ${ }^{1-4}$ Our group further demonstrated the size sensitivity for carbon-nitrogen ring opening reactions. ${ }^{5}$ Catalytic activity and selectivity dependence on nanocrystal shape has also been demonstrated. For instance, both cyclohexene and cyclohexane formed on cuboctahedral nanocrystals during benzene hydrogenation / dehydrogenation while only cyclohexane formed on cubic nanocrystals. ${ }^{6}$ These observations were consistent with previous results for $\mathrm{Pt}$ single-crystal studies. In order to further investigate the roles of nanocrystal size and shape in transition metal heterogeneous catalysis, it is essential to simultaneously control the size and the shape of the nanocrystals.

The well-defined (100) facets on Pt nanocube surfaces make the Pt nanocubes an ideal material for understanding the influence of nanocrystal facets on catalytic performance. ${ }^{6,7}$ Several synthetic methods for cubic Pt nanocrystals were reported previously, however, sizes of these shaped nanocrystals were typically around $10 \mathrm{~nm}$ or larger. ${ }^{7-10}$ Sub-10 nm nanocrystals demonstrate different physical and chemical properties from large nanocrystals. ${ }^{11-18}$ Previous research unambiguously showed that the structure dependency of activity and selectivity are more significant for nanocrystals smaller than 10 $\mathrm{nm} .^{3-5}$ Also, smaller nanocrystals have higher dispersion than larger ones and this fact permits more efficient use of precious metals. The lack of a synthetic method for shaped nanocrystals in single-digit nanometer range reflects that size reduction while maintaining the shape is challenging. The difficulty is partly because the shape controlled nanocrystals are normally kinetically stable, but not thermodynamically stable. Therefore, change of any synthetic factor that reduces the size also causes shape change. ${ }^{19}$ The development of a 
general strategy for simultaneous control of size and shape is critical for synthesis of nanocrystals with shape control in the single-digit nanometer range.

In this work, we report a facile method to synthesize sub-10 nm Pt nanopolyhedra and nanocubes dominantly enclosed in six (100) facets. The size and the shape of these sub-10 nm Pt nanocrystals were both tuned by simply controlling the reducing rate of metal precursors at different stages of the synthesis. Nanocubes with tunable size from 5 to $9 \mathrm{~nm}$ and nanopolyhedra with size of 3.5 and $5.0 \mathrm{~nm}$ were synthesized and then supported into an inert mesoporous silica support (MCF-17) for shape and size dependent catalytic studies. The Pt nanocrystals with different size and shape displayed similar turnover frequencies for ethylene hydrogenation, which was consistent with previous studies ${ }^{3,4}$ on $\mathrm{Pt}$ nanocrystals and single crystals. Normalized per mass of Pt, the activities of the $5 \mathrm{~nm}$ nanopolyhedra and nanocubes were higher than that of the $9 \mathrm{~nm}$ nanocubes, which demonstrated that the smaller Pt nanocubes were advantageous compared to the larger nanocubes due to its higher surface-to-bulk ratio. Shape dependent selectivity was clearly demonstrated for the nanopolyhedra and nanocubes by pyrrole hydrogenation. The dominant product over the Pt nanocubes was $n$-butylamine. Over Pt nanopolyhedra of similar size, a mixture of pyrrolidine and $n$-butylamine formed and this selectivity difference represents a significant result in shape controlled selectivity. In order to further understand this shape sensitivity, sum-frequency generation (SFG) surface vibrational spectra of pyrrole adsorbed onto Pt single crystals were compared. The study showed that, consistent with our catalytic results, $n$-butylamine formation is enhanced on the Pt (100) surface, the dominant surface of the nanocubes, relative to the Pt (111) surface.

\section{Experiment section}


Chemicals. Triblock copolymer Pluronic P123 (Alderich), 1,3,5-trimethylbenzene (Sigma-Aldrich), tetraethyl orthosilicate $\left(\mathrm{Si}\left(\mathrm{OC}_{2} \mathrm{H}_{5}\right)_{4}\right.$, Sigma-Aldrich), ammonium fluoride (Sigma-Aldrich), chloroplatinic acid hexahydrate $\left(\mathrm{H}_{2} \mathrm{PtCl}_{6} \cdot 6 \mathrm{H}_{2} \mathrm{O}, \geq 37.50 \% \mathrm{Pt}\right.$ basis, Sigma-Aldrich), ammonium hexachloroplatinate (IV) $\left(\left(\mathrm{NH}_{4}\right)_{2} \mathrm{PtCl}_{6}, 99 \%\right.$; Sigma-Aldrich), ammonium tetrachloroplatinate (II) $\left(\left(\mathrm{NH}_{4}\right)_{2} \mathrm{PtCl}_{4}, 99 \%\right.$; Sigma-Aldrich), poly(vinylpyrrolidone) ( $\mathrm{PVP}, M \mathrm{w}=29,000 ;$ Sigma-Aldrich), teramethylammonium bromide $\left(\mathrm{N}^{+}\left(\mathrm{CH}_{3}\right)_{4} \mathrm{Br}\right.$, $99 \%$; Sigma-Aldrich), ethylene glycol (>98\%; EMD), and solvents (analytical grade) including acetone, ethanol, hexanes and chloroform were used without further purification.

Sub-10 nm Pt nanocrystal synthesis. A total $0.05 \mathrm{mmol}$ of $\mathrm{Pt}$ ions $\left(\mathrm{NH}_{4}\right)_{2} \mathrm{Pt}(\mathrm{IV}) \mathrm{Cl}_{6}$ and $\left.\left(\mathrm{NH}_{4}\right)_{2} \mathrm{Pt}(\mathrm{II}) \mathrm{Cl}_{4}\right), 0.75 \mathrm{mmol}$ tetramethylammonium bromide, and $1.00 \mathrm{mmol}$ poly(vinylpyrrolidone) (in terms of the repeating unit; M.W. 29,000) were dissolved into $10 \mathrm{~mL}$ ethylene glycol in a $25 \mathrm{~mL}$ round bottom flask at room temperature. The same results were obtained if $\left(\mathrm{NH}_{4}\right)_{2} \mathrm{Pt}(\mathrm{IV}) \mathrm{Cl}_{6}$ was replaced by $\mathrm{H}_{2} \mathrm{Pt}(\mathrm{IV}) \mathrm{Cl}_{6} \cdot 6 \mathrm{H}_{2} \mathrm{O}$ as the $\mathrm{Pt}(\mathrm{IV})$ ion source. The $\mathrm{Pt}(\mathrm{IV})$ to total $\mathrm{Pt}(\mathrm{Pt}(\mathrm{II})+\mathrm{Pt}(\mathrm{IV}))$ molar ratios were $0,20 \%, 50 \%, 80 \%$, and $100 \%$ for synthesis of $3.5 \mathrm{~nm}$ nanopolyhedra, $5 \mathrm{~nm}$ nanocubes, $6 \mathrm{~nm}$ nanocubes, $7 \mathrm{~nm}$ nanocubes, and $9 \mathrm{~nm}$ nanocubes respectively. The mixed solution was heated to $180{ }^{\circ} \mathrm{C}$ in an oil bath at $60{ }^{\circ} \mathrm{C} / \mathrm{min}$. For $5 \mathrm{~nm}$ Pt spherical nanopolyhedra, the $\mathrm{Pt}(\mathrm{IV})$ to $\mathrm{Pt}$ $(\operatorname{Pt}(\mathrm{II})+\mathrm{Pt}(\mathrm{IV}))$ molar ratio was 0.2 and the temperature was $140^{\circ} \mathrm{C}$. The solutions were held at these respective temperatures for 20 min under argon protection and magnetic stirring, resulting in a dark brown solution. After the solution was cooled to room temperature, acetone $(90 \mathrm{~mL})$ was then added to form a cloudy black suspension which was separated by centrifugation at 3,000 rpm for $10 \mathrm{~min}$. The black product was collected 
by discarding the colorless supernatant. The products were further washed three times by precipitation/dissolution (re-dispersed in $20 \mathrm{~mL}$ of ethanol and then precipitated by adding $80 \mathrm{~mL}$ of hexanes). The nanocrystals were then re-dispersed in $10 \mathrm{~mL}$ ethanol for characterization and catalyst preparation.

MCF-17 synthesis. The synthesis of MCF-17 followed the previous reports. ${ }^{20,21}$ In a typical preparation, $4 \mathrm{~g}$ of 1,3,5-trimethylbenzene (TMB) was added into a $75 \mathrm{~mL}$ aqueous solution that contained $4 \mathrm{~g}$ of triblockcopolymer Pluronic P123 and $10 \mathrm{~mL}$ of concentrated $\mathrm{HCl}$. The solution was stirred at $40^{\circ} \mathrm{C}$ for $2 \mathrm{~h}$ and $9.2 \mathrm{~mL}$ of tetraethoxysilane (TEOS) was then added. After stirring for 5 min, the solution was aged at $40^{\circ} \mathrm{C}$ for $20 \mathrm{~h}$ under a quiescent condition. Then, $46 \mathrm{mg}$ of $\mathrm{NH}_{4} \mathrm{~F}$ was added and the solution was transferred to an autoclave and was aged at $100^{\circ} \mathrm{C}$ for another $24 \mathrm{~h}$. The precipitate product was filtered, washed with water and ethanol, and calcined in air at $600{ }^{\circ} \mathrm{C}$ for $6 \mathrm{~h}$. The white powder was kept in desiccator for further use.

Pt on MCF-17 catalysts preparation. Pt colloidal solution $(1 \mathrm{mg} / \mathrm{mL})$ was diluted by ethanol $(0.1 \mathrm{mg} / \mathrm{mL})$. The desired amount of solution $(0.25 \mathrm{wt}$. \% Pt) was added to mesoporous MCF-17 and sonicated for $3 \mathrm{~h}$ at room temperature by a commercial ultrasonic cleaner (Branson, 1510R-MT, $70 \mathrm{~W}, 42 \mathrm{kHz}$ ). The brown precipitates were separated by centrifugation (3000 rpm, $20 \mathrm{~min}$ ), thoroughly washed with ethanol two more times, and dried in an oven at $373 \mathrm{~K}$ overnight. ${ }^{22}$

Characterization Methods. Shape, size and lattice structure of the nanocrystals were analyzed using a FEI Tecnai G2 S-Twin transmission electron microscope (TEM) and Philips CM200/FEG high-resolution TEM (HRTEM), operated at $200 \mathrm{kV}$. X-ray photoelectron spectroscopy (XPS) experiments were performed on a Perkin-Elmer PHI 
5300 XPS spectrometer with a position-sensitive detector and a hemispherical energy analyzer in an ion-pumped chamber (evacuated to $2 \times 10^{-9}$ Torr). The $\mathrm{Al}-\mathrm{K} \alpha(\mathrm{BE}=1486.6$ eV) X-ray source was operated at $300 \mathrm{~W}$ with $15 \mathrm{kV}$ acceleration voltage. Binding energies (BE) were calibrated by setting the measured $\mathrm{BE}$ of C $1 \mathrm{~s}$ to $285 \mathrm{eV}$. Elemental analyses by inductively coupled plasma atomic emission spectroscopy (ICP-AES) were conducted at Galbraith Laboratories, Inc. (Knoxville, TN). Dispersions were determined at $303 \mathrm{~K}$ by $\mathrm{H}_{2}$ chemisorption (Autosorb I, Quantachrome Instruments) using the total isotherm.

Ethylene and pyrrole hydrogenation. For catalytic studies, samples were diluted with low surface area quartz and loaded into glass reactors. Temperature was controlled by a PID controller (Watlow 96) and a type-K thermocouple. Gas flows (all from Praxair and UHP) were regulated using calibrated mass flow controllers. Before the reaction, samples were reduced in $50 \mathrm{~mL} \min ^{-1}$ of 76 torr of $\mathrm{H}_{2}$ with a He balance for $1 \mathrm{~h}$ at $100^{\circ} \mathrm{C}$. For ethylene hydrogenation, the gases were 10 torr of ethylene, 100 torr of $\mathrm{H}_{2}$ with a balance of He. For pyrrole hydrogenation, the feed was 4 torr of pyrrole (Sigma-Aldrich, $>98 \%$ ) and 400 torr of $\mathrm{H}_{2}$ with a balance of $\mathrm{He}$. The desired partial pressure of pyrrole was achieved by bubbling He through pyrrole and assuming saturation. For both reactions, gas composition was analyzed with flame ionization (FID) and thermal conductivity (TCD) detectors on a HP 5890 Series II gas chromatograph (GC). Turnover frequencies were determined by normalizing the conversion to the number of Pt surface atoms determined by hydrogen chemisorption.

Sum-frequency generation (SFG) surface vibrational spectroscopy study. For SFG measurements, an active/passive mode-locked Nd:YAG laser (Leopard D-20, Continuum) with a pulse width of $20 \mathrm{ps}$ and a repetition rate of $20 \mathrm{~Hz}$ was used. The 
fundamental output at $1064 \mathrm{~nm}$ was sent through an optical parametric generation/amplification (OPA/OPG) stage where a tunable IR (2300-4000 $\left.\mathrm{cm}^{-1}\right)$ and a second-harmonic VIS $(532 \mathrm{~nm})$ beam were created. The IR $(150 \mu \mathrm{J})$ and VIS $(200 \mu \mathrm{J})$ beams were spatially and temporally overlapped on the crystal surface at angles of incidence of $55^{\circ}$ and $60^{\circ}$, respectively, with respect to the surface normal. The generated SFG beam was collected and sent through a monochromator to a photomultiplier tube to detect the SFG signal intensity.

\section{Results and Discussions}

Synthesis and characterization of shaped sub-10 $\mathrm{nm}$ Pt nanocrystals. The addition of foreign metal ions is effective for controlling the shape of metallic nanocrystals. Several Fe or Ag ion mediated syntheses of shape controlled Pt nanocrystals have been reported. ${ }^{9,23,24}$ However, the role of the foreign metal ions is not clear and the final state of the foreign metal is hard to be confirmed. Moreover, in the Ag-mediated synthesis, the catalytic activity is greatly affected by the presence of foreign ions even though the foreign ion was barely detectable. ${ }^{25}$ In the present approach, bromide ions were used as the shape directing agent. ${ }^{26-30}$ The bromide-mediated strategy is more favorable catalytically because the bromide species are only on the nanocrystal surface and are easily removed after synthesis by washing. In general, the metal salts ( $\mathrm{Pt}(\mathrm{IV})$ and $\mathrm{Pt}(\mathrm{II})$ ), the surfactants (PVP), and the shape directing agent (bromide ions) were dissolved in ethylene glycol at room temperature to form a precursor solution. Previous studies have shown that the bromide species selectively adsorbed onto Pt (100) crystal faces and induced the formation of $\mathrm{Pt}$ nanocubes. ${ }^{7,8}$ The mixed precursor solution was then rapidly heated to the desired temperature $\left(140\right.$ and $180^{\circ} \mathrm{C}$ for nanopolyhedra and nanocubes, respectively) and 
maintained at this temperature for 20 min with vigorous stirring. The formation of $9(8.8 \pm$ 1.2) $\mathrm{nm}$ (edge) Pt nanocubes ( $85 \%$ cubes, $15 \%$ tetrahedra or irregular crystals) is revealed by the transmission electron microscopy (TEM) images (Figure 1a) when the $\operatorname{Pt}(\mathrm{IV})$ salt $\left(\left(\mathrm{NH}_{4}\right)_{2} \mathrm{PtCl}_{6}\right)$ was used in the precursor solution. High resolution (HR) TEM image indicates that the Pt nanocubes are single crystals and enclosed by six (100) faces (Figure 1b). During the synthesis, the solution instantly turned brown after 2 min of heating and then turned darker with increasing time. By monitoring the crystal growth process with TEM (Figure 2), we hypothesized that the $9 \mathrm{~nm}$ Pt nanocube growth was a two-stage process. During the initial stage, the Pt nuclei (around $3.5 \mathrm{~nm}$ ) formed rapidly in the solution (Figure 2a). In the second stage, the remaining Pt ions were reduced onto these nuclei with preferential growth direction along the [111] direction because bromide ions stabilized the (100) faces (Figure $2 \mathrm{~b}$ and c). If this assumption is true, smaller nanocubes should form by increasing the number of the nuclei in the initial stage and decreasing the remaining Pt ions in the second stage. To demonstrate this concept, we introduced $\mathrm{Pt}(\mathrm{II})$ salt $\left(\left(\mathrm{NH}_{4}\right)_{2} \mathrm{PtCl}_{4}\right)$, which can be reduced more easily than $\mathrm{Pt}(\mathrm{IV})$, to partially replace the Pt(IV) salt in the initial solution. By doing so, the number of nuclei in the initial stage increased without changing the kinetics of the second stage (Scheme 1). TEM images show the monodispersity and well-defined shape of these Pt nanocubes synthesized by altering the concentration ratios of $\mathrm{Pt}(\mathrm{II})$ to $\mathrm{Pt}(\mathrm{IV}$ ) in the initial solution (Figure 1c, e, and g). Pt nanocubes with sizes of $7(6.9 \pm 1.8) \mathrm{nm}, 6(5.9 \pm 0.7) \mathrm{nm}$, and $5(5.0 \pm 0.4) \mathrm{nm}$ were obtained when the ratios of $\mathrm{Pt}(\mathrm{IV})$ to total $\mathrm{Pt}$ ions (referred to as $\mathrm{Pt}(\mathrm{IV})$ ratio in the following) was $0.8,0.5$, and 0.2 , respectively. The high yield and well-defined shape of these sub- $10 \mathrm{~nm}$ nanocubes synthesized by this method were clearly revealed by TEM and 
HRTEM. Regardless of size, the yields of cubic nanocrystals are more than $80 \%$. HRTEM images revealed that these nanocubes with different sizes are all enclosed by six (100) faces (Figure 1d, f, and h). The observation of mainly [100] direction orientated nanocrystals (more then $70 \%$ of $\sim 50$ measured nanocrystals in each size) under HRTEM without any sample tilting indicated the high yield and well-defined shape. Size control of the Pt nanocrystals is demonstrated as a function of the Pt(IV) ratios in Figure 3. When all the Pt (IV) ions were replaced by Pt (II) ions in the initial solution, the $3.5(3.5 \pm 0.3) \mathrm{nm}$ single-crystalline Pt nanocrystals were obtained (Figure 1k and 1), which are similar to the nucleus nanocrystals (Figure 2a) that were observed in the initial stage of $9 \mathrm{~nm}$ nanocubes synthesis. We believe that these $3.5 \mathrm{~nm}$ single-crystalline nanocrystals were also formed by reducing Pt (II) ion in the syntheses of $5 \mathrm{~nm}, 6 \mathrm{~nm}$, and $7 \mathrm{~nm}$ single-digit nanocubes. These rapidly formed nanocrystals served as nuclei in the synthesis and the increase of these nuclei density significantly helps to reduce the size of the nanocubes.

The shape of these sub-10 $\mathrm{nm}$ nanocrystals was tuned by changing the growth rate. Nanocubes are kinetically stabilized by bromide ions while the growth rate of the second stage can be reduced via decreasing the reaction temperature. Then, the more thermodynamically stable polyhedral nanocrystals were obtained rather than cubic nanocrystals (Scheme 1). ${ }^{19}$ For example, $5 \mathrm{~nm}$ single-crystalline $\mathrm{Pt}$ spherical nanopolyhedra were synthesized while the Pt(IV) ratio was 0.2 and the temperature was $140^{\circ} \mathrm{C}$ (Figure 1i and $\mathrm{j}$ ). The much slower color change of the solution during the synthesis at $140^{\circ} \mathrm{C}$ indicated the slower reducing rate of Pt ions. The Pt nanopolyhedra oriented randomly under TEM grids and the [111] orientated crystals were observed more frequently due to the larger lattice space (Figure 1j). 
The oxidation state of these nanocrystals was measured by X-ray photoelectron spectroscopy (XPS). A thin layer of washed nanocrystals was deposited on a single-crystal silicon wafer for XPS and the $\mathrm{Pt} 4 \mathrm{f}_{7 / 2}$ peaks of different samples were compared. The XPS results revealed that all the nanocrystals are mainly in metallic state, regardless of the size and shape (Table 1). The small amount of oxidized Pt $(<20 \%)$ was contributed by a thin layer of oxidized Pt on the surfaces. Only trace amounts of bromide ions were detected. The ratios of bromide ions to total $\mathrm{Pt}$ atoms were below 10\%. The amounts of oxidized $\mathrm{Pt}$ and bromide ions both increased with decreasing nanocrystal size, which correlated to a higher surface-to-bulk ratio. This trend supported that oxidized Pt and bromide ions are mainly on the surfaces of the nanocrystals. This one-pot synthetic strategy allowed the synthesis of shape-controlled nanocrystals without foreign metal ions and also the continuous size control in the single-digit nanometer regime. The ability to continuously tune the size of shape controlled nanocrystals in this regime is very important for studying catalytic properties and is the focus of the rest of this contribution.

Loading Pt onto MCF-17 mesoporous silica. To study the catalysis over the Pt nanocrystals, ethylene and pyrrole hydrogenation over the $9 \mathrm{~nm}$ nanocubes, $5 \mathrm{~nm}$ nanocubes, and $5 \mathrm{~nm}$ nanopolyhedra supported on the 3D inert mesoporous silica support were studied. To promote homogeneous dispersion of the Pt nanocrystals within the silica pores, the mesoporous silica support with large pores, MCF-17, were used. The surface area and pore volume of the MCF-17 are $500 \mathrm{~m}^{2} \mathrm{~g}^{-1}$ and $2.5 \mathrm{~cm}^{3} \mathrm{~g}^{-1}$, respectively. Compared to SBA-15, a more popular mesoporous silica support for metal nanoparticles ${ }^{3,4,31}$, MCF-17 has a larger pore size (diameter $\sim 30-50 \mathrm{~nm}$ ) and a broader connection window size ( $\sim 17 \mathrm{~nm}$ ), which easily permitted loading of the large Pt nanocrystals. The hydrophilic 
surfaces of the nanocrystals also helped the loading onto the hydrophilic metal oxide supports. Mesoporous MCF-17 powder was added to ethanol solutions containing nanocrystals and sonicated for $3 \mathrm{~h}$. Without sonication, nanocrystals were mainly on the external surface of MCF-17 and might form agglomeration easily during reactions. ${ }^{4}$ After sonication, the precipitates were separated by centrifugation and thoroughly washed by ethanol. The successful loading is indicated by the clear supernatants after centrifugation. The loading of all samples was confirmed by TEM and elemental analysis. As shown in Figure 4, the $5 \mathrm{~nm}$ Pt nanocubes, $5 \mathrm{~nm}$ nanopolyhedra, and $9 \mathrm{~nm}$ Pt nanocubes were homogeneously distributed in the MCF-17. The actual loading of Pt was confirmed to be 0.2 wt. \% (0.25 wt. \% nominal loading) for all samples by inductively coupled plasma (ICP) spectrometry (Table 2). Only small amount of Pt was lost during the loading and thorough washing processes and it further demonstrated the advantage of using MCF-17 as support. The amounts of Pt surface atoms were determined by hydrogen chemisorption (Table 2). Dispersions are near what are expected based on size arguments so it suggests an accessible active surface of the Pt nanocrystals. The catalysts were reduced in 76 torr of $\mathrm{H}_{2}$ with a $\mathrm{He}$ balance for $1 \mathrm{~h}$ at $100^{\circ} \mathrm{C}$ before chemisorption and catalytic experiments.

Catalytic activity for ethylene hydrogenation. The catalytic activities for ethylene hydrogenation are summarized in Table 2. Regardless of size and shape, TOFs were $5 \pm 1$ $\mathrm{s}^{-1}$ and these values correlate well to rates obtained over single crystals. ${ }^{32}$ Since it is structure insensitive reaction, the similarities indicated that calculated number of catalytic sites was correct and the synthetic additives used to control shape and size were effectively removed by washing. Simultaneous size control is highlighted by normalizing ethane formation by the mass of Pt. Then, the activity of the $5 \mathrm{~nm}$ single-digit nanocrystal was 
much higher than that of the $9 \mathrm{~nm}$ nanocrystals. This result unambiguously revealed the advantage of the smaller nanocrystals. The number of active sites was significantly increased, without sacrificing the shape, when the size of the nanocrystals decreased in the single-digit nanometer region.

Pyrrole hydrogenation selectivity. Aromatic ring hydrogenation is an ideal reaction for selectivity studies as it has been investigated extensively on Pt single crystal. ${ }^{33-35}$ In this study, selectivity dependency of these nanocubes and nanopolyhedra was demonstrated using pyrrole hydrogenation (Scheme 2). Because it has been shown that the ring opening of pyrrole to $n$-butylamine is structure sensitive ${ }^{5}$, it is suitable for probing shape-dependent reaction selectivity in catalysis. Also, this reaction is an important model reaction for fuel processing involving removal of $\mathrm{N}$ from $\mathrm{N}$-containing organics. In Figure 5, the structure sensitivity for hydrogenation of pyrrole to pyrrolidine, $n$-butylamine, and butane is presented at equal conversions at each given temperature, which is necessary for selectivity comparisons. The shape of the nanocrystals led to clear selectivity differences. For both 5 $\mathrm{nm}$ and $9 \mathrm{~nm} \mathrm{Pt}$ nanocubes, $n$-butylamine was the dominant product at all studied temperatures. For the $5 \mathrm{~nm}$ nanopolyhedra, however, pyrrolidine (up to 30\%) and n-butylamine were both formed at lower temperatures.

In order to study this structure sensitive selectivity and determine the intermediates on the surfaces of nanocubes, the sum-frequency generation (SFG) surface vibrational spectroscopy was performed to study the adsorption species of pyrrole hydrogenation over Pt (100) single crystal. Under the catalytic reaction condition, SFG is a much more effective technique for interfacial chemical analysis than conventional linear spectroscopy, such as infrared absorption or Raman. ${ }^{36-41}$ In linear spectroscopy, the absorption is mainly 
contributed by bulk gases, and therefore the absorption generated by adsorbed species at the catalyst surface is relatively small. In contrast, due to the second-order nonlinear optical process of SFG, the absorption of isotropic bulk gases do not appear in the SFG spectrum and the absorption of adsorbed species, the symmetry of which is broken at the surface, is solely enhanced. Most importantly, previous SFG study ${ }^{35}$ and NEXAFS study ${ }^{42}$ have shown that the reaction intermediates (pyrroline, pyrrolidine, and $n$-butylamine) of pyrrole hydrogenation bind through the nitrogen to the metal surface in an upright orientation on Pt single crystal surface (Scheme 2) and can be recorded by SFG. The SFG spectrum recorded during pyrrole hydrogenation over Pt (100) single crystal is shown in Figure 5. By comparing the spectra of $\mathrm{Pt}(111)^{35}$ and $\mathrm{Pt}(100)$ single crystals for pyrrole hydrogenation at the same temperature $(298 \mathrm{~K})$, we observed that the $\mathrm{CH}_{2}$ group bands contributed by disorder in the butyl chains of $n$-butylamine and the $\mathrm{CH}_{3}$ group bands on $\mathrm{Pt}$ (100) single crystal were relatively stronger than the ones on Pt (111) single crystal. The result suggested that the amount of $n$-butylamine on the Pt surface was greatly enhanced on the (100) crystal surface. Based on this observation, the shape controlled selectivity of pyrrole hydrogenation should be caused by the hydrogenation of pyrrolidine to $n$-butylamine being promoted by the Pt (100) surface. Since the Pt nanocubes are mainly enclosed in (100) surfaces, pyrrolidine was converted into $n$-butylamine on the surface of the nanocubes. Therefore, our nanocubes promote the formation of $n$-butylamine at lower temperatures. Compared to the (100) surface rich nanocubes, the polyhedral nanocrystals contain more thermodynamically stable (111) surfaces, which inhibit turnover of $n$-butylamine. Detailed descriptions of SFG theory and peak assignment can be found elsewhere ${ }^{35}$ and detailed SFG studies on single-digit nanocrystals for further understanding 
the mechanism of the selectivity are currently under investigation. There are two important points should be highlighted in this selectivity behavior study. First, the selectivity difference between the 3D mesoporous material supported Pt nanopolyhedra and nanocubes was significant and agreed with the single crystal studies. Second, the comparable selectivity for pyrrole hydrogenation over the $5 \mathrm{~nm}$ and $9 \mathrm{~nm}$ single-digit $\mathrm{Pt}$ nanocubes revealed that the (100) crystal surfaces were still dominant for these nanocubes with size as small as $5 \mathrm{~nm}$. It clearly indicated that we successfully decreased the size of the nanocubes to the single-digit nanometer region without sacrificing the well-defined surface structure.

Conclusion. Sub-10 nm Pt nanocubes and nanopolyhedra with tunable size were synthesized by controlling the reducing rate of Pt ions in our one-pot synthetic system. This synthetic method allowed us to synthesize Pt nanocrystals with tunable size and shape, which are ideal for catalysis studies. Ethylene hydrogenation rates over the mesoporous silica supported Pt nanocrystals indicated similar catalytic activities as compared to Pt single crystals, which suggested a clean and accessible catalytic surface. The nanocubes showed significant product selectivity difference from the nanopolyhedra during pyrrole hydrogenation due to the well-controlled crystal surfaces. These observations clearly indicate the importance of size and shape control of nanocrystals in catalytic research.

Acknowledgment. This work was supported by the Director, Office of Science, Office of Basic Energy Sciences, Division of Chemical Sciences, Geological and Biosciences and Division of Materials Sciences and Engineering of the U.S. Department of Energy under Contract No. DE-AC02-05CH11231. We also thank the Molecular Foundry 
of the Lawrence Berkeley National Laboratory for using their facilities and Professor A.

Paul Alivisatos for use of the TEM. 


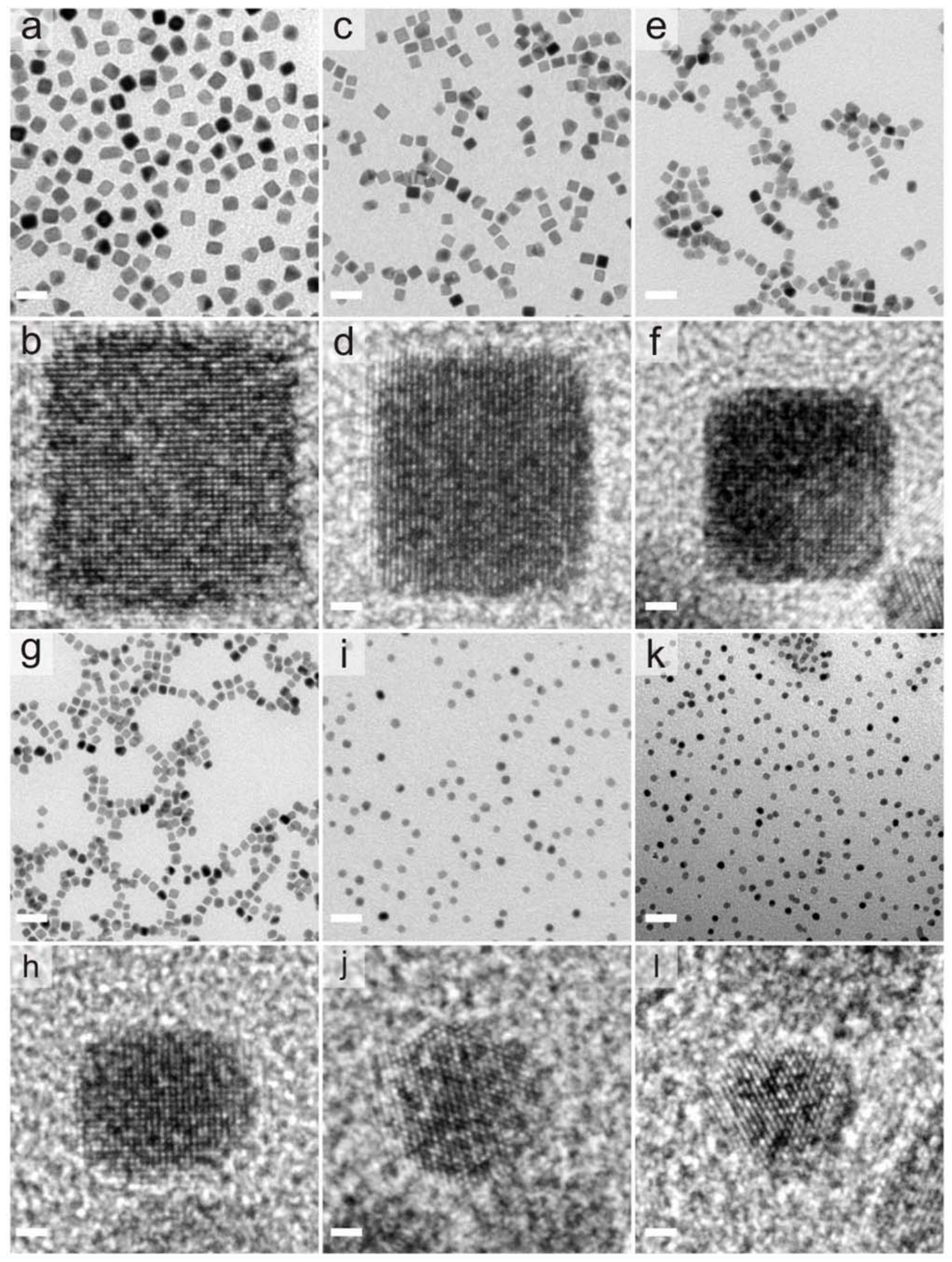

Figure 1. TEM and HRTEM images of Pt nanocrystals with different shape and size. TEM 
images of a) $9 \mathrm{~nm}$ nanocubes, c) $7 \mathrm{~nm}$ nanocubes, e) $6 \mathrm{~nm}$ nanocubes, g) $5 \mathrm{~nm}$ nanocubes, i) $5 \mathrm{~nm}$ nanopolyhedra, and k) $3.5 \mathrm{~nm}$ nuclei. HRTEM images of a single b) $9 \mathrm{~nm}$ nanocube, d) $7 \mathrm{~nm}$ nanocube, f) $6 \mathrm{~nm}$ nanocube, and h) $5 \mathrm{~nm}$ nanocube along the [100] zone axis. HRTEM images of a single j) $5 \mathrm{~nm}$ nanopolyhedron, and l) $3.5 \mathrm{~nm}$ nucleus along the [111] zone axis. The scale bars are $20 \mathrm{~nm}$ in the TEM images and $1 \mathrm{~nm}$ in the HRTEM images. 

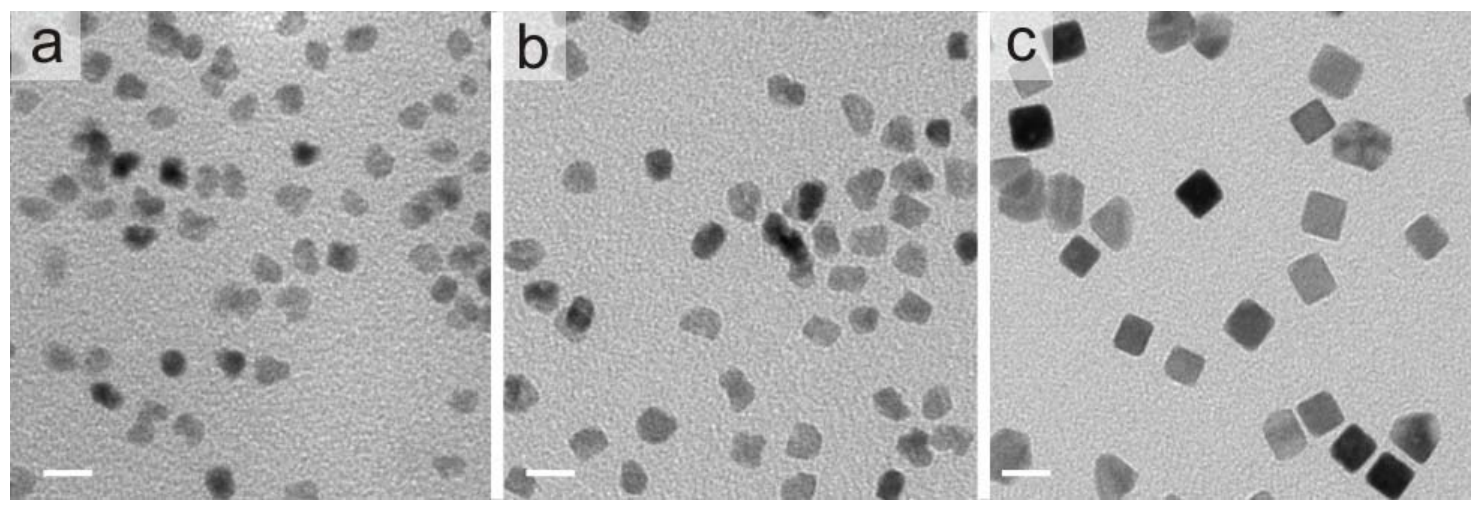

Figure 2. TEM images of $9 \mathrm{~nm}$ Pt nanocubes in different stages of the synthesis. a) $2 \mathrm{~min}$, b) $5 \mathrm{~min}$, and c) $10 \mathrm{~min}$. The scale bars are $10 \mathrm{~nm}$ in the TEM images 


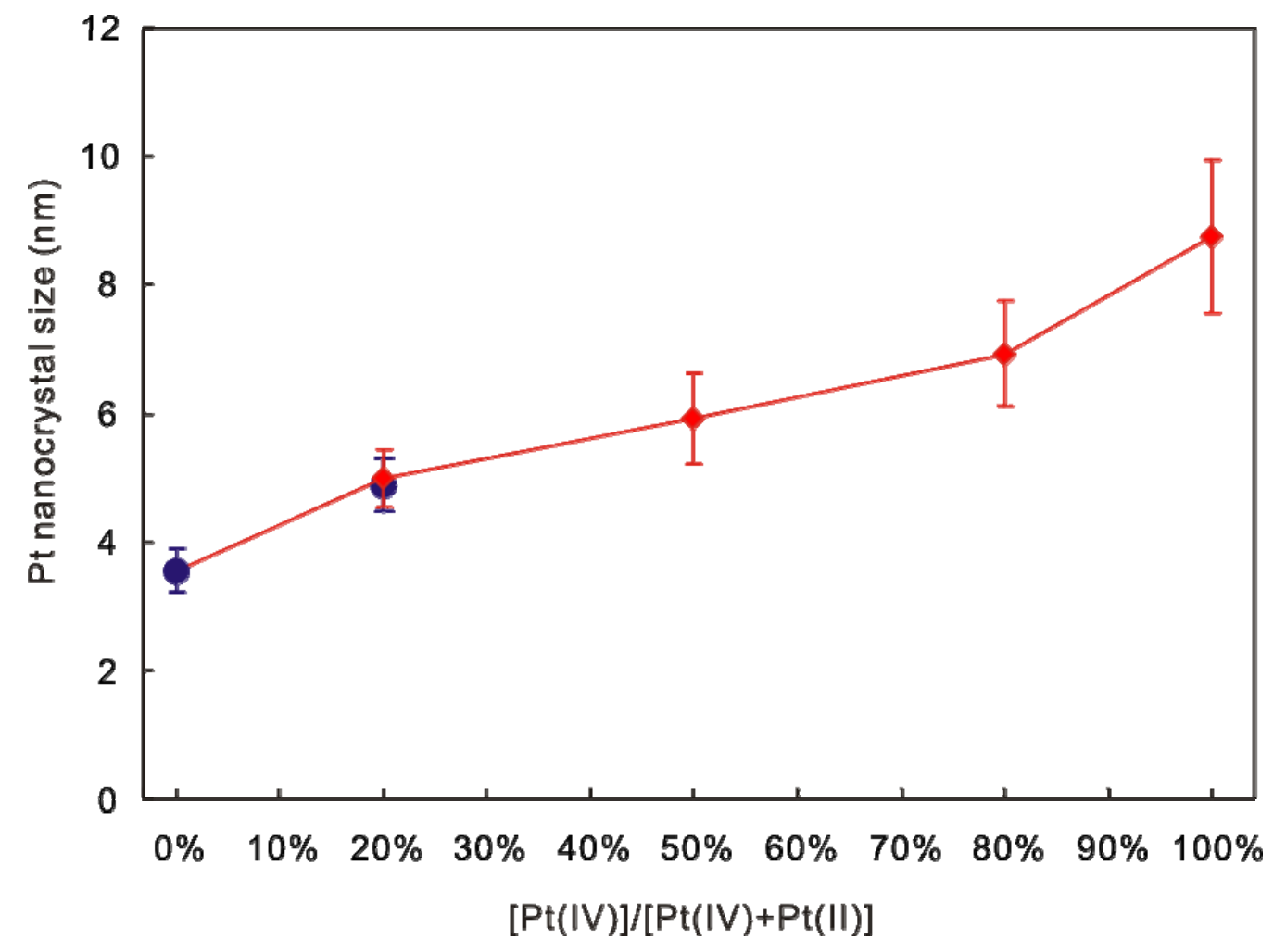

Figure 3. The size of Pt nanocrystals as a function of [Pt(IV) to Pt(IV) $+\mathrm{Pt}(\mathrm{II})]$ in the initial solution. The blue circles represent nanopolyhedra and the red diamonds represent nanocubes. 
Scheme 1. Schematic illustration of the size and shape control of sub-10 nm Pt nanocrystals. The size and the shape of the nanocrystals are controlled by reduction rate kinetics during the two stages.

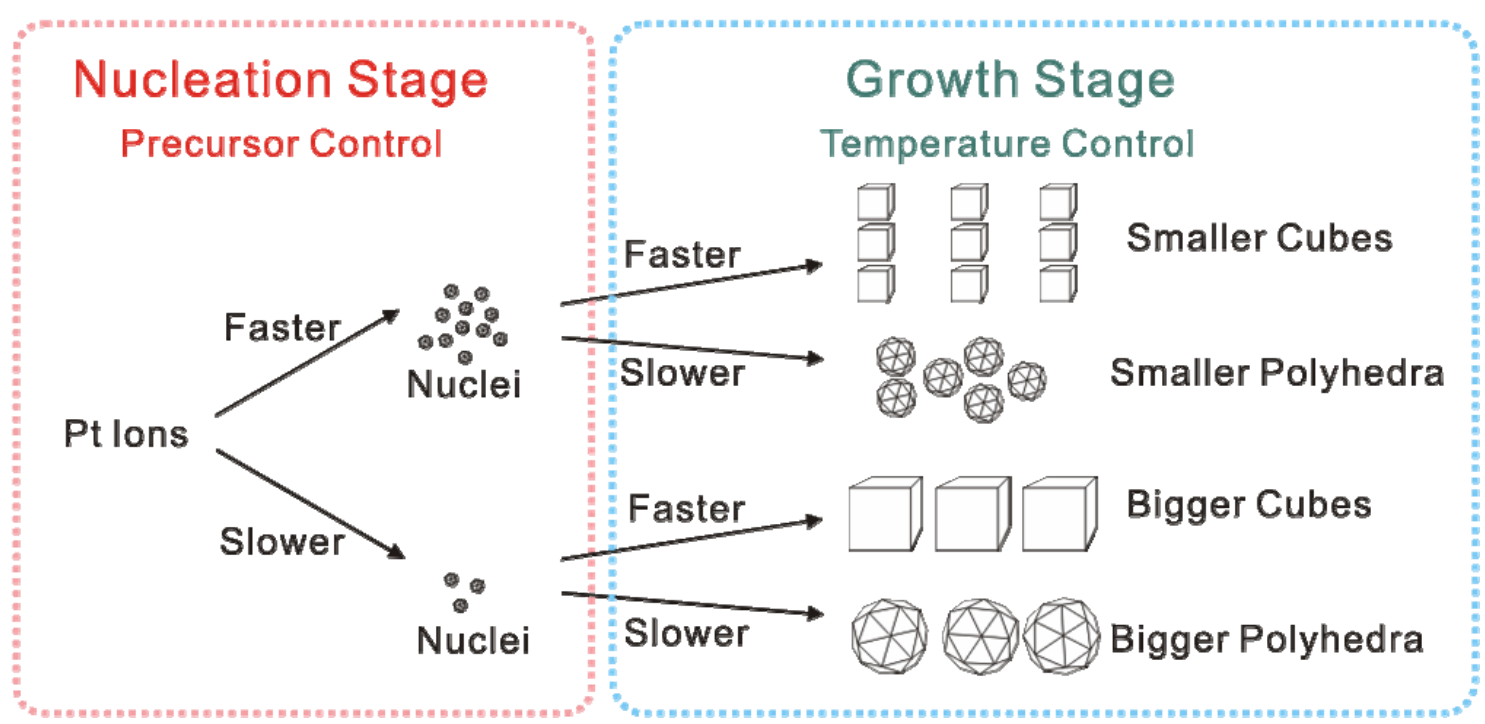



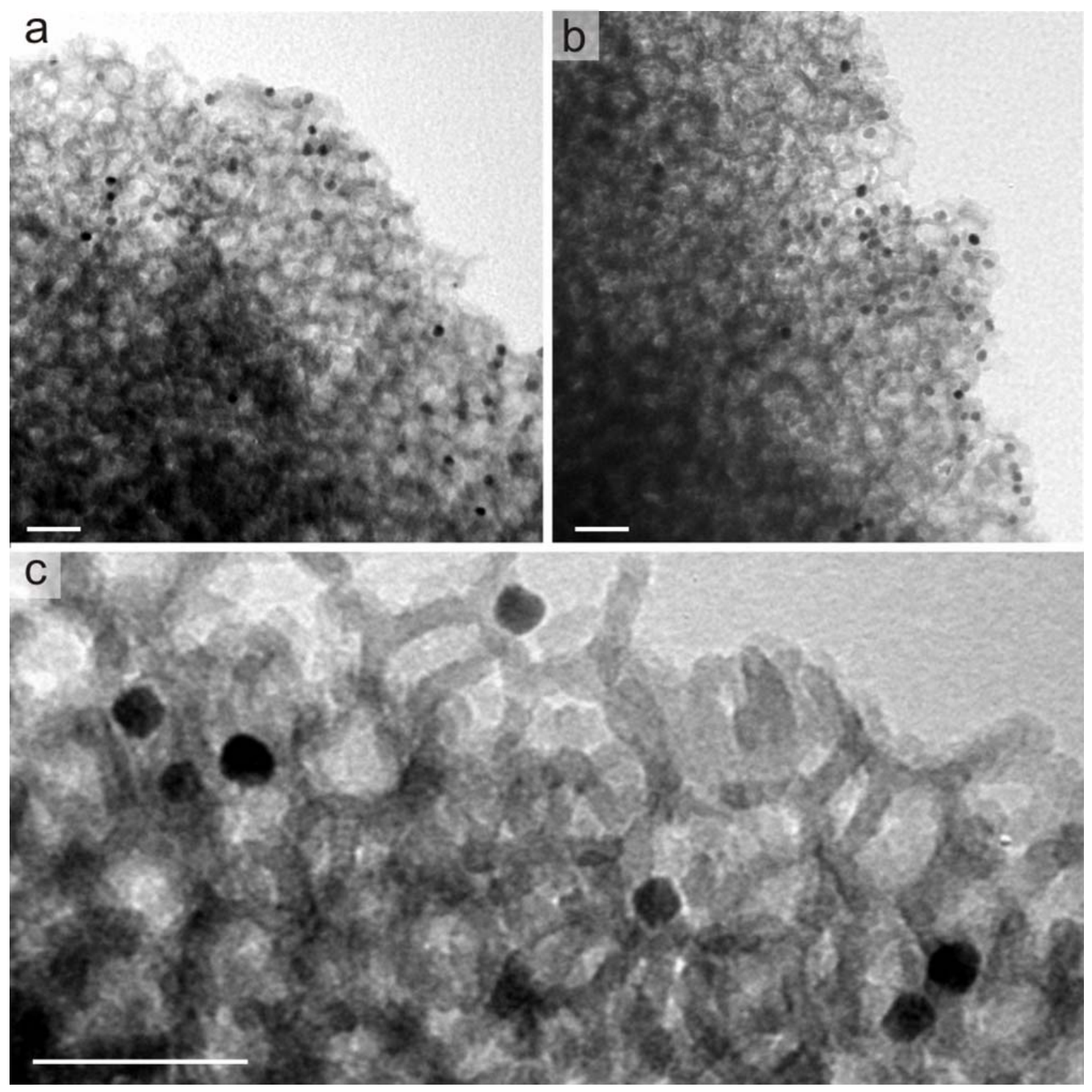

Figure 4. TEM images of Pt nanocrystals loaded onto mesoporous MCF-17, a) $5 \mathrm{~nm}$ nanocubes, b) $5 \mathrm{~nm}$ nanopolyhedra, and c) $9 \mathrm{~nm}$ nanocubes. The scale bars are $100 \mathrm{~nm}$ in the TEM images. 
Table 1. X-ray photoelectron spectra results of Pt nanocrystals.

\begin{tabular}{lccc}
\hline & Metallic Pt & Oxidized Pt & $\mathrm{Br} /$ Total Pt \\
\hline $9 \mathrm{~nm}$ Pt nanocubes & $88.3 \%$ & $11.7 \%$ & $0.9 \%$ \\
$7 \mathrm{~nm}$ Pt nanocubes & $87.5 \%$ & $12.6 \%$ & $1.0 \%$ \\
$6 \mathrm{~nm}$ Pt nanocubes & $88.5 \%$ & $11.5 \%$ & $3.7 \%$ \\
$5 \mathrm{~nm}$ Pt nanocubes & $85.6 \%$ & $14.4 \%$ & $5.2 \%$ \\
$5 \mathrm{~nm}$ Pt nanopolyhedra & $83.4 \%$ & $16.7 \%$ & $9.9 \%$ \\
\hline
\end{tabular}

Table 2. Ethylene hydrogenation rates and kinetic parameters on MCF-17 supported Pt nanocrystals.

\begin{tabular}{|c|c|c|c|c|c|}
\hline Catalyst $^{a}$ & Loading $^{b}$ & Dispersion $^{c}$ & $\begin{array}{c}\text { Activity } \\
\left(\mathrm{mmol} \mathrm{C}_{2} \mathrm{H}_{6} \mathrm{~g}_{\mathrm{Pt}}^{-1} \mathrm{~s}^{-1}\right)\end{array}$ & $\begin{array}{l}\text { TOF } \\
\left(\mathrm{s}^{-1}\right)^{d}\end{array}$ & $\begin{array}{c}\mathrm{Ea} \\
\left(\mathrm{kcal} \mathrm{mol}^{-1}\right)\end{array}$ \\
\hline $\begin{array}{c}5.0 \mathrm{~nm} \mathrm{Pt} \\
\text { Nanopolyhedra }\end{array}$ & $0.2 \%$ & 0.19 & 5.3 & 5.5 & 7.4 \\
\hline $\begin{array}{c}5.0 \mathrm{~nm} \mathrm{Pt} \\
\text { Nanocubes }\end{array}$ & $0.2 \%$ & 0.20 & 6.2 & 6.0 & 7.2 \\
\hline $\begin{array}{c}9.0 \mathrm{~nm} \mathrm{Pt} \\
\text { Nanocubes }\end{array}$ & $0.2 \%$ & 0.08 & 2.0 & 5.0 & 8.0 \\
\hline $\operatorname{Pt}(111)^{e}$ & N/A & N/A & N/A & 6.1 & 10.8 \\
\hline
\end{tabular}


Scheme 2. Pyrrole hydrogenation network and proposed molecular adsorption over a Pt surface.
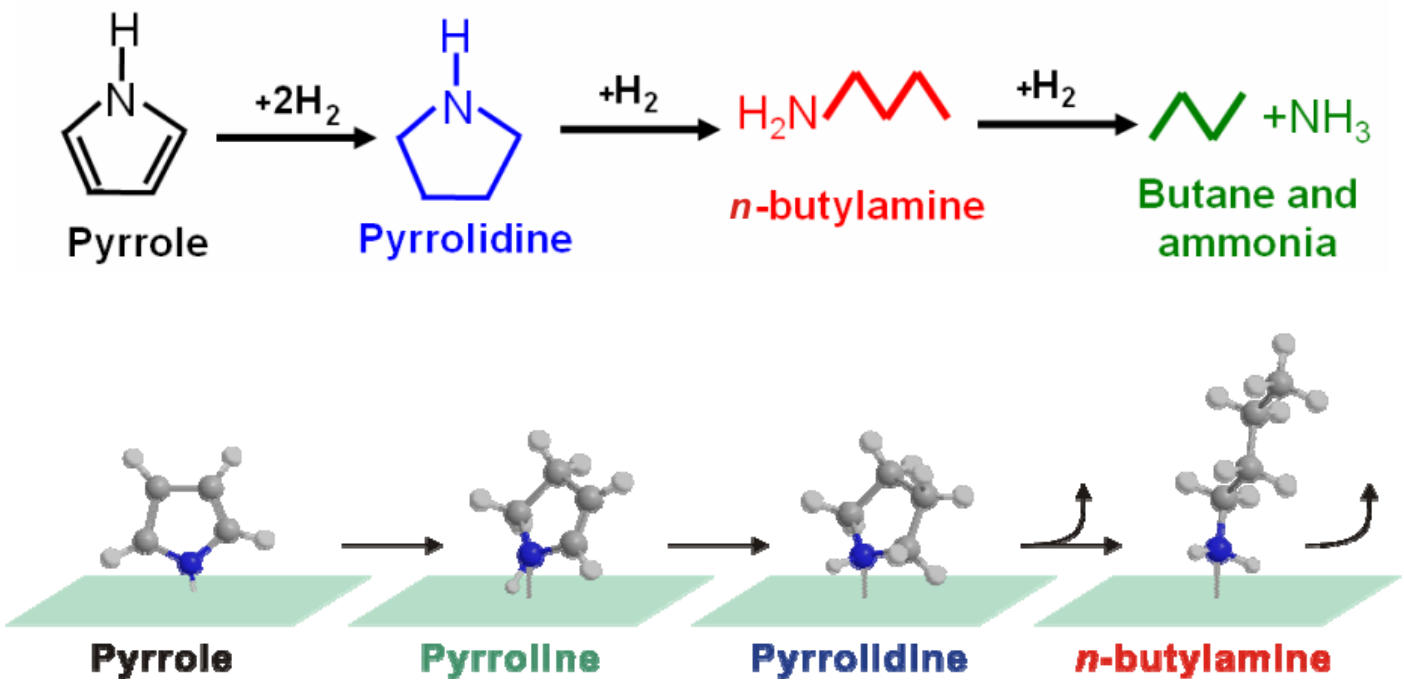


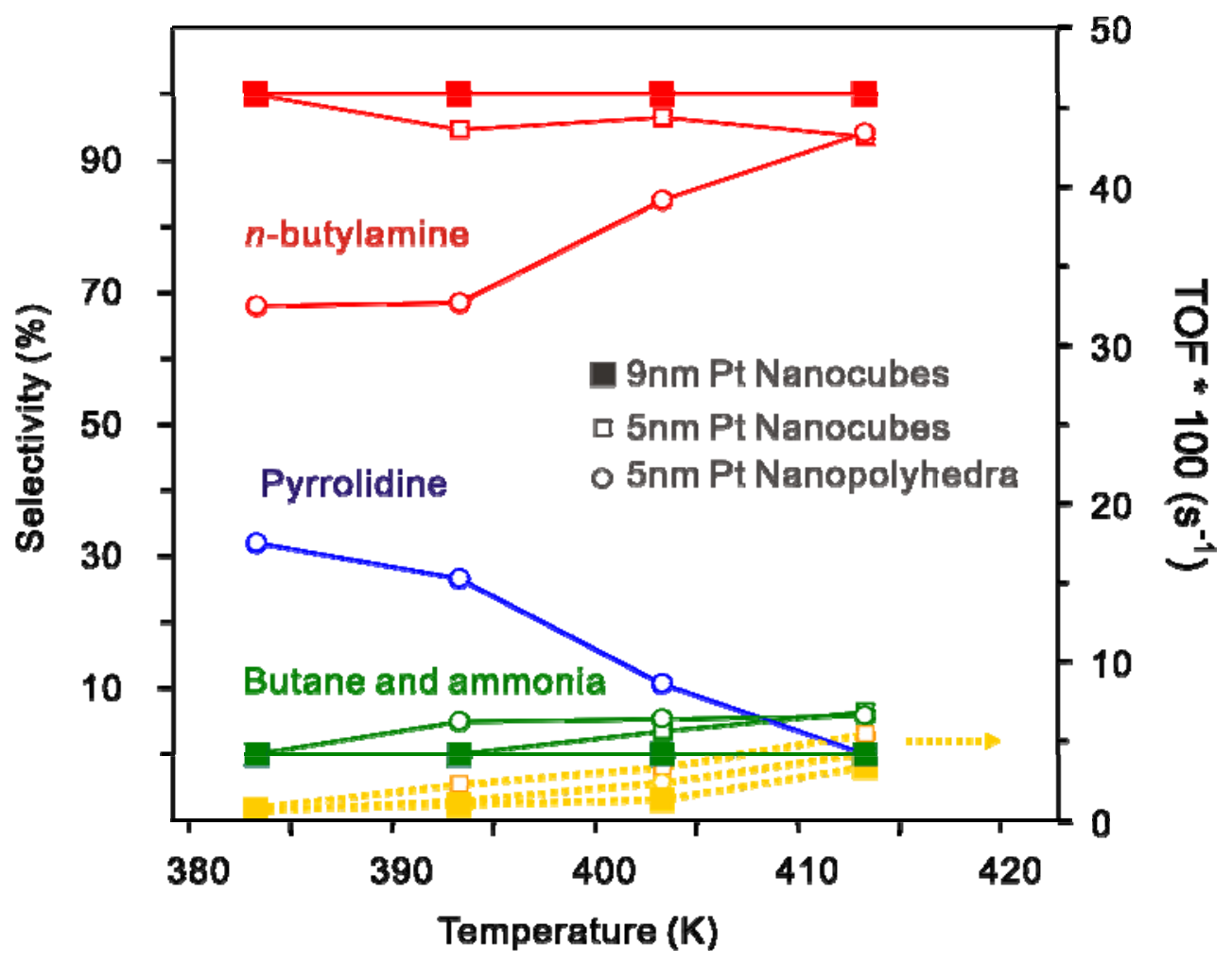

Figure 5. Pyrrole hydrogenation selectivity for Pt nanocrystals with different size and shape as a function of temperature. The color of the line coordinated to Scheme 2. Solid squares, open squares, and open spheres represent $9 \mathrm{~nm}$ nanocubes, $5 \mathrm{~nm}$ nanocubes, and 5 $\mathrm{nm}$ nanopolyhedra, respectively. Feed was 4 torr of pyrrole and 400 torr of $\mathrm{H}_{2}$ with a $\mathrm{He}$ balance. 


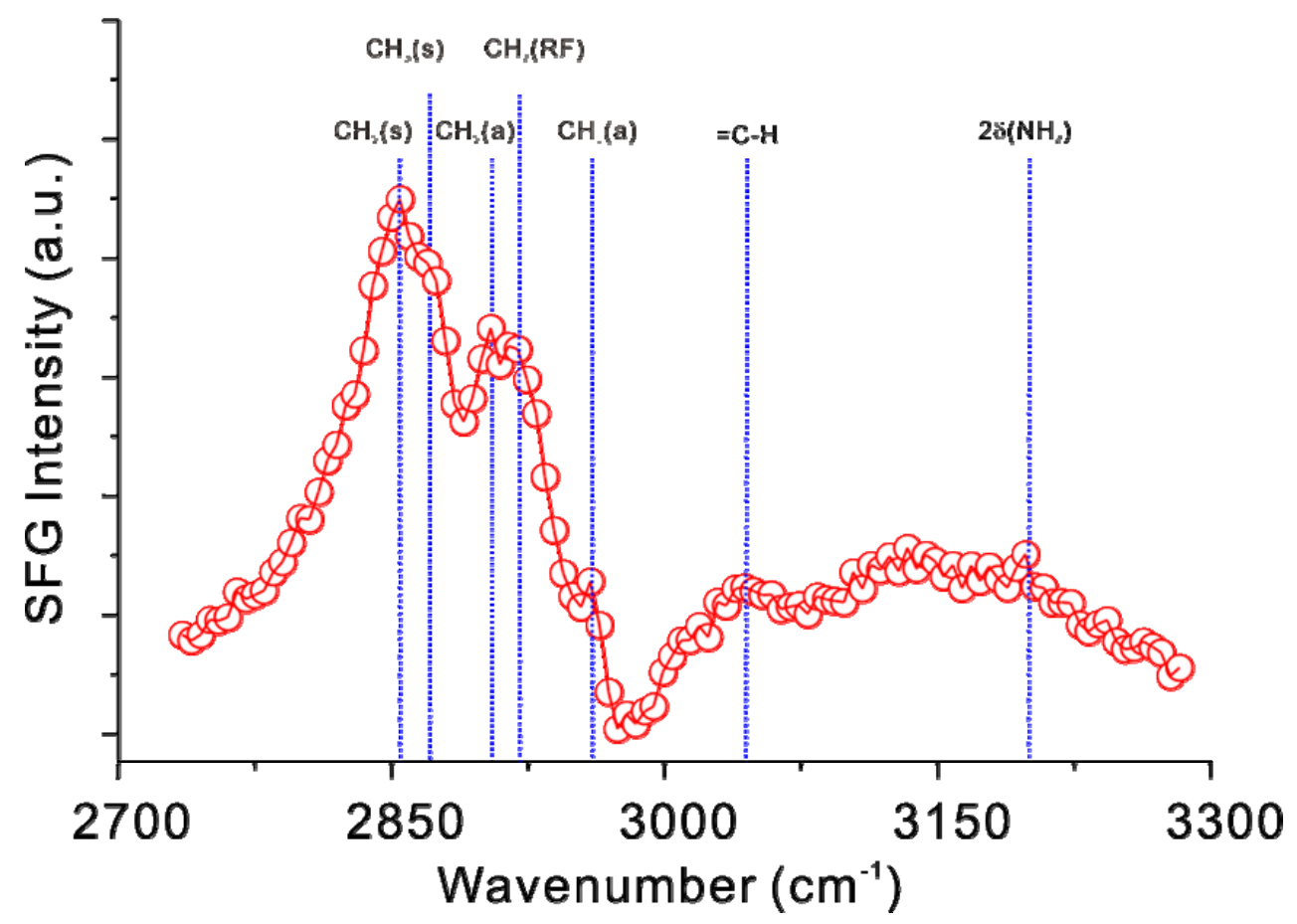

Figure 6. SFG vibrational spectra recorded during pyrrole hydrogenation (3 Torr pyrrole, 30 Torr $\mathrm{H}_{2}$ ) over $\mathrm{Pt}(100)$ at $298 \mathrm{~K}$. The peaks from butylamine dominate the spectrum. $\mathrm{CH}_{2}(\mathrm{FR})$ represents Fermi resonance of the methyl group. 


\section{REFERENCES}

(1) Martin, G. A. Journal of Catalysis 1979, 60, 452.

(2) Carter, J. L.; Cusumano, J. A.; Sinfelt, J. H. Journal of Physical Chemistry 1966, 70, 2257.

(3) Song, H.; Rioux, R. M.; Hoefelmeyer, J. D.; Komor, R.; Niesz, K.; Grass, M.; Yang, P. D.; Somorjai, G. A. Journal of the American Chemical Society 2006, $128,3027$.

(4) Rioux, R. M.; Song, H.; Hoefelmeyer, J. D.; Yang, P.; Somorjai, G. A. Journal of Physical Chemistry B 2005, 109, 2192.

(5) Kuhn, J. N.; Huang, W.; Tsung, C.-K.; Zhang, Y.; Somorjai, G. A. 2008; Vol. 130, p 14026.

(6) Bratlie, K. M.; Lee, H.; Komvopoulos, K.; Yang, P. D.; Somorjai, G. A. Nano Letters 2007, 7, 3097.

(7) Lee, H.; Habas, S. E.; Kweskin, S.; Butcher, D.; Somorjai, G. A.; Yang, P. D. Angewandte Chemie-International Edition 2006, 45, 7824.

(8) Zhang, Y. W.; Grass, M. E.; Kuhn, J. N.; Tao, F.; Habas, S. E.; Huang, W. Y.; Yang, P. D.; Somorjai, G. A. Journal of the American Chemical Society 2008, $130,5868$.

(9) Song, H.; Kim, F.; Connor, S.; Somorjai, G. A.; Yang, P. D. Journal of Physical Chemistry B 2005, 109, 188.

(10) Ren, J. T.; Tilley, R. D. Journal of the American Chemical Society 2007, 129, 3287.

(11) Whetten, R. L.; Khoury, J. T.; Alvarez, M. M.; Murthy, S.; Vezmar, I.; Wang, Z. L.; Stephens, P. W.; Cleveland, C. L.; Luedtke, W. D.; Landman, U. Advanced Materials 1996, 8, 428.

(12) Daniel, M. C.; Astruc, D. Chemical Reviews 2004, 104, 293.

(13) Boettcher, S. W.; Strandwitz, N. C.; Schierhorn, M.; Lock, N.; Lonergan, M. C.; Stucky, G. D. Nature Materials 2007, 6, 592.

(14) Zheng, N. F.; Stucky, G. D. Chemical Communications 2007, 3862.

(15) Shipway, A. N.; Katz, E.; Willner, I. Chemphyschem 2000, 1, 18.

(16) Wang, Z. L. Journal of Physical Chemistry B 2000, 104, 1153.

(17) Alivisatos, A. P.; Johnsson, K. P.; Peng, X. G.; Wilson, T. E.; Loweth, C. J.; Bruchez, M. P.; Schultz, P. G. Nature 1996, 382, 609.

(18) Ahmadi, T. S.; Wang, Z. L.; Green, T. C.; Henglein, A.; ElSayed, M. A. Science 1996, 272, 1924.

(19) Tao, A. R.; Habas, S.; Yang, P. D. Small 2008, 4, 310.

(20) Han, Y.; Lee, S. S.; Ying, J. Y. Chemistry of Materials 2007, 19, 2292.

(21) Schmidt-Winkel, P.; Lukens, W. W.; Yang, P. D.; Margolese, D. I.; Lettow, J. S.; Ying, J. Y.; Stucky, G. D. Chemistry of Materials 2000, 12, 686.

(22) Lettow, J. S.; Han, Y. J.; Schmidt-Winkel, P.; Yang, P. D.; Zhao, D. Y.; Stucky, G. D.; Ying, J. Y. Langmuir 2000, 16, 8291.

(23) Wang, C.; Daimon, H.; Onodera, T.; Koda, T.; Sun, S. H. Angewandte Chemie-International Edition 2008, 47, 3588. 
(24) Wang, C.; Daimon, H.; Lee, Y.; Kim, J.; Sun, S. Journal of the American Chemical Society 2007, 129, 6974.

(25) Grass, M. E.; Yue, Y.; Habas, S. E.; Rioux, R. M.; Teall, C. I.; Yang, P.; Somorjai, G. A. Journal of Physical Chemistry C 2008, 112, 4797.

(26) Wiley, B. J.; Chen, Y. C.; McLellan, J. M.; Xiong, Y. J.; Li, Z. Y.; Ginger, D.; Xia, Y. N. Nano Letters 2007, 7, 1032.

(27) Xiong, Y. J.; Cai, H. G.; Wiley, B. J.; Wang, J. G.; Kim, M. J.; Xia, Y. N. Journal of the American Chemical Society 2007, 129, 3665.

(28) Tsung, C. K.; Kou, X. S.; Shi, Q. H.; Zhang, J. P.; Yeung, M. H.; Wang, J. F.; Stucky, G. D. Journal of the American Chemical Society 2006, 128, 5352.

(29) Jana, N. R.; Gearheart, L.; Murphy, C. J. Journal of Physical Chemistry B 2001, $105,4065$.

(30) Jana, N. R.; Gearheart, L.; Murphy, C. J. Chemical Communications 2001, 617.

(31) Zhao, D. Y.; Huo, Q. S.; Feng, J. L.; Chmelka, B. F.; Stucky, G. D. Journal of the American Chemical Society 1998, 120, 6024.

(32) Zaera, F.; Somorjai, G. A. Journal of the American Chemical Society 1984, 106, 2288.

(33) Bratlie, K. M.; Montano, M. O.; Flores, L. D.; Paajanen, M.; Somorjai, G. A. Journal of the American Chemical Society 2006, 128, 12810.

(34) Bratlie, K. M.; Kliewer, C. J.; Somorjai, G. A. Journal of Physical Chemistry B 2006, 110, 17925.

(35) Kliewer, C. J.; Bieri, M.; Somorjai, G. A. Journal of Physical Chemistry C 2008, 112, 11373.

(36) Bratlie, K. M.; Komvopoulos, K.; Somorjai, G. A. Journal of Physical Chemistry C 2008, 112, 11865.

(37) Bratlie, K. M.; Somorjai, G. A. Journal of Physical Chemistry C 2007, 111, 6837.

(38) Kweskin, S. J.; Rioux, R. M.; Habas, S. E.; Komvopoulos, K.; Yang, P.; Somorjai, G. A. Journal of Physical Chemistry B 2006, 110, 15920.

(39) Yang, M.; Somorjai, G. A. Journal of the American Chemical Society 2004, 126, 7698.

(40) Somorjai, G. A.; Rupprechter, G. Journal of Physical Chemistry B 1999, 103, 1623.

(41) Cremer, P. S.; Su, X. C.; Shen, Y. R.; Somorjai, G. A. Journal of the American Chemical Society 1996, 118, 2942.

(42) Tourillon, G.; Raaen, S.; Skotheim, T. A.; Sagurton, M.; Garrett, R.; Williams, G. P. Surface Science 1987, 184, L345. 


\section{DISCLAIMER}

This document was prepared as an account of work sponsored by the United States Government. While this document is believed to contain correct information, neither the United States Government nor any agency thereof, nor The Regents of the University of California, nor any of their employees, makes any warranty, express or implied, or assumes any legal responsibility for the accuracy, completeness, or usefulness of any information, apparatus, product, or process disclosed, or represents that its use would not infringe privately owned rights. Reference herein to any specific commercial product, process, or service by its trade name, trademark, manufacturer, or otherwise, does not necessarily constitute or imply its endorsement, recommendation, or favoring by the United States Government or any agency thereof, or The Regents of the University of California. The views and opinions of authors expressed herein do not necessarily state or reflect those of the United States Government or any agency thereof or The Regents of the University of California. 